Jean CÉSAR ${ }^{1,2}$

JérémY BOUYER ${ }^{1,3}$

Laurent GRANJON ${ }^{4}$

Massouroudini AKoudJIN ${ }^{1,5}$

Dominique LouPPE ${ }^{6}$

${ }^{1}$ Centre international de recherche-développement sur l'élevage

en zone subhumide

BP 454, Bobo-Dioulasso

Burkina Faso

2 Cirad

Isra-Lnerv

Urp Pôle pastoral zones sèches BP 2057, Dakar Hann

Sénégal

${ }^{3}$ Cirad

Umr Contrôle des maladies animales exotiques et émergentes Campus international de Baillarguet

34398 Montpellier Cedex 5

France

4 Ird

Umr 022 Cbgp

Campus de Bel-Air

BP 1386, Dakar, CP 18524

Sénégal

5 Institut de développement rural Université polytechnique de Bobo-Dioulasso

BP 1091, Bobo-Dioulasso

Burkina Faso

6 Cirad

Ur B\&sef

Campus international

de Baillarguet

34398 Montpellier Cedex 5

France

\section{Les relictes forestières de la falaise de Banfora : les dégradations au voisinage de Bobo-Dioulasso, Burkina Faso}

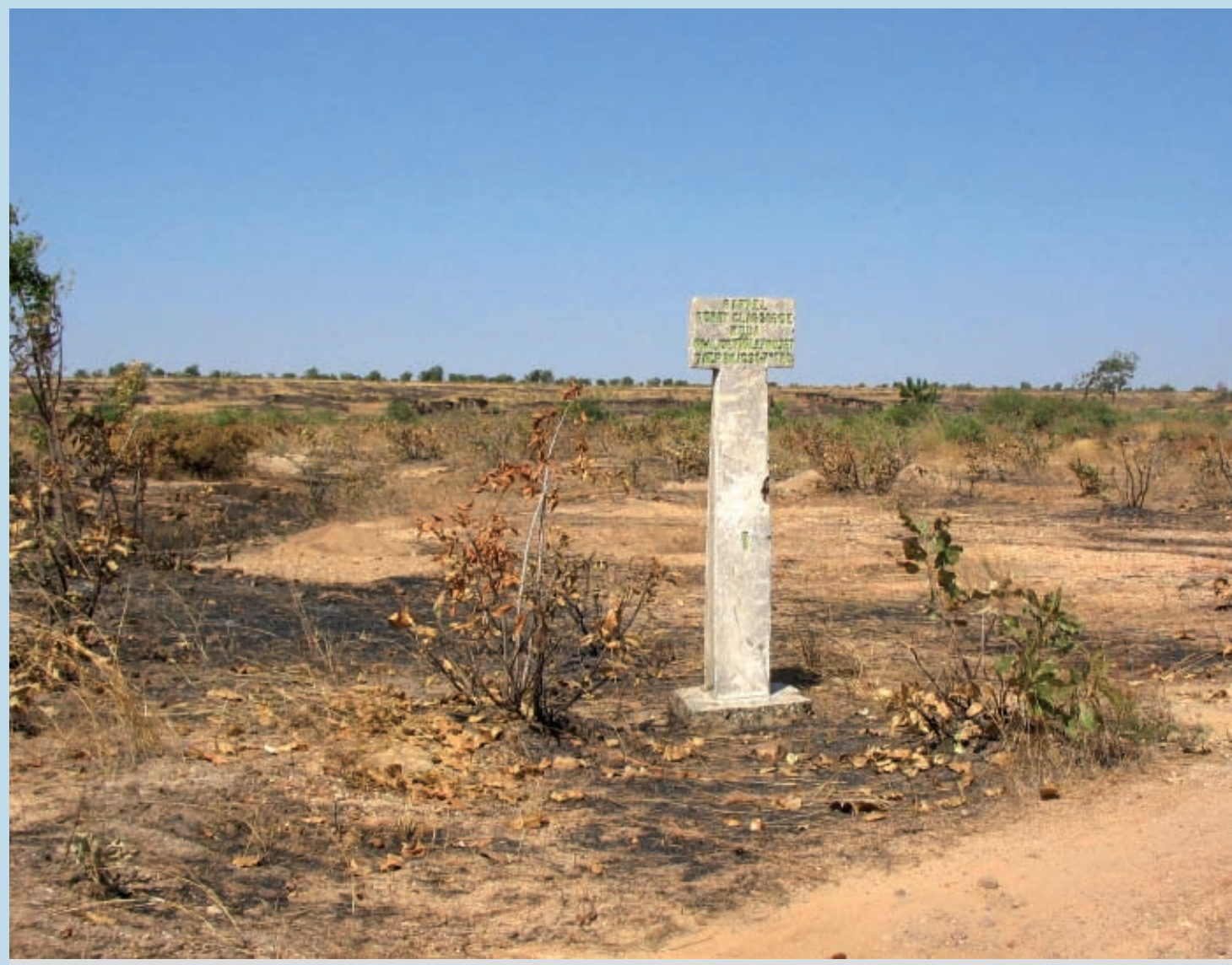

Photo 1.

La forêt classée de Koua, projet du $7^{\text {e }}$ Fed. Photo J. César. 


\section{RÉSUMÉ}

LES RELICTES FORESTIÈRES

DE LA FALAISE DE BANFORA : LES DÉGRADATIONS AU VOISINAGE DE BOBO-DIOULASSO, BURKINA FASO

Le suivi de la végétation et l'inventaire de bioindicateurs animaux, micromammifères et insectes, a permis de dresser un bilan des dégradations environnementales dans trois villages situés à proximité de Bobo-Dioulasso. Les dégâts apparemment les plus graves résultent de la coupe des arbres pour le bois de feu et de l'extraction minière du sable et du gravier pour les constructions. Ces activités sont la conséquence du développement urbain. Les dégradations environnementales dues à l'agriculture et à l'élevage paraissent moins importantes. L'ensemble de ces activités humaines fait que les terres maraîchères de Koro sont en train de disparaître, tout comme la forêt classée de Koua. Les suivis et inventaires, réalisés pour mieux cerner cette évolution anthropique de l'environnement, montrent que les espèces animales réagissent à cette forte perturbation du milieu amplifiée par les phénomènes fréquents d'érosion. En conclusion de cette étude, des mesures de protection et de reboisement sont proposées pour limiter l'érosion dans les champs et sur les berges de la rivière. L'extraction minière des matériaux de construction pourrait aussi être organisée pour moins impacter l'environnement ; mais cela risque d'être difficile à mettre en œuvre car les intérêts individuels priment face aux besoins collectifs.

Mots-clés : falaise gréseuse, bioindicateur, micromammifères, insecte, dégradation environnementale, extraction minière.

\section{ABSTRACT}

\section{RELICT FORESTS ON THE \\ BANFORA CLIFFS: ENVIRONMENTAL DEGRADATION AROUND BOBO- DIOULASSO IN BURKINA FASO}

Vegetation monitoring and inventories of animal, micro-mammal and insect bioindicators were used to assess environmental degradation in three villages close to the town of Bobo-Dioulasso. The damage that appeared most severe resulted from tree felling for fuelwood and from sand and gravel extraction for building. Both activities are a consequence of urban development. Environmental degradation due to crop and livestock farming seems less severe. However, all of these human pressures are damaging Koro's market gardens as well as the Koua protected forest. The monitoring and inventory work now under way to deepen understanding of human-induced environmental changes shows that animal species are reacting to the disruption of their habitats, which is worsened by frequent erosion. The study concludes with proposals for protective measures and reforestation to curb erosion in crop fields and along river banks. The extraction of building materials also needs to be organised to reduce its impact on the environment, but this is likely to be problematical as long as individual interests take priority over collective needs.

Keywords: sandstone cliffs, bioindicator, smallmammals, insect, environmental degradation, mineral extraction.

\section{RESUMEN}

\section{LOS BOSQUES RELICTOS DEL ACANTILADO DE BANFORA: LAS DEGRADACIONES EN LAS CERCANÍAS DE BOBO-DIOULASSO, BURKINA FASO}

El seguimiento de la vegetación y el inventario de bioindicadores animales, micromamíferos e insectos, permitió establecer un balance de las degradaciones ambientales en tres pueblos ubicados en las proximidades de BoboDioulasso. Aparentemente, los daños más graves provienen de la corta de árboles para leña y de la extracción minera de arena y grava para las construcciones. Estas actividades son consecuencia del desarrollo urbano. Las degradaciones ambientales causadas por la agricultura y ganadería parecen menos importantes. El conjunto de estas actividades humanas hacen que estén desapareciendo las tierras de huertas de Koro, al igual que el bosque protegido de Koua. Los seguimientos e inventarios, realizados para identificar mejor esta evolución antrópica del medio ambiente, muestran que las especies animales reaccionan a esta fuerte perturbación del medio amplificada por los frecuentes fenómenos de erosión. A modo de conclusión, en este estudio se proponen medidas de protección y reforestación para limitar la erosión en los campos y las riberas del río. La extracción minera de los materiales de construcción también podría organizarse para reducir su impacto ambiental; pero esto puede que sea difícilmente aplicable ya que los intereses individuales prevalecen sobre las necesidades colectivas.

Palabras clave: acantilado de arenisca, bioindicador, micromamíferos, insecto, degradación ambiental, extracción minera. 


\section{Introduction}

Le Projet Fsp (Fonds de solidarité prioritaire) 2002-87 «Gestion durable des ressources sylvo-pastorales et production fourragère dans l'Ouest du Burkina-Faso », financé par l'ambassade de France à Ouagadougou, avait permis de réaliser une première étude sur l'origine des peuplements végétaux et animaux des falaises de Banfora (CÉSAR et al., 2010). Cette étude concluait à la grande richesse écologique des falaises et à la nécessité de les protéger. À partir de là, il s'est agi d'évaluer les risques de dégradation de ces ressources en appréciant les niveaux actuels de perturbations. Cette évaluation environnementale avait pour objectif ultime d'aider les populations à prendre en charge la gestion de leur patrimoine naturel.

Un bilan des dégradations environnementales a été établi dans les villages de Koro, Kuinima et Kouakoualé pour initier la protection de leurs relictes forestières. Ces villages sont situés entre 10 et 12 kilomètres $(\mathrm{km})$ au Sud-Est de Bobo-Dioulasso, vers l'extrémité Nord de la falaise de Banfora (figure 1). Ces villages sont contigus et se partagent le site cultuel de Dafra, la relicte forestière la plus remarquable et la plus fréquentée par les habitants de BoboDioulasso. Ce lieu de sacrifices rituels est aussi un site touristique visité pour la beauté de sa gorge encaissée, sa végétation et ses silures géants. Alors que Kuinima, déjà partiellement inclus dans l'agglomération de BoboDioulasso, s'étend sur le plateau au-dessus de la falaise, Koro et Kouakoualé sont au pied de celle-ci.

Les travaux d'écologie débouchent sur la constatation de graves dégradations ; ils sont complétés par des propositions pour améliorer la protection des ressources biologiques.

\section{Matériel et méthodes}

Koro, Kouakoualé et Kuinima sont des villages proches de Bobo-Dioulasso, avec des activités périurbaines (production laitière, maraîchère et fruitière) développées autour des sources de la falaise gréseuse.

\section{Le diagnostic écologique}

Le diagnostic écologique a été réalisé en deux étapes. La première est un inventaire des sites remarquables, soit pour leur intérêt écologique, soit pour leur état de dégradation. La seconde est une étude plus scientifique de détail destinée à mettre en évidence l'évolution des écosystèmes.

\section{Choix des sites}

Le choix des sites a été déterminé d'abord en s'appuyant sur des images satellitaires (Landsat 7 Enhanced Thematic Mapper, d'octobre 1999), puis sur des villageois intéressés par les questions environnementales. Sur quatorze sites pressentis, quatre, dont le site sacré de Dafra, ont été retenus en accord avec les représentants villageois, pour y réaliser les études de détail sur la végétation et la faune.

\section{Les études écologiques}

L'objectif est de déterminer le sens et la vitesse d'évolution de quelques formations végétales types soumises aux effets des différents facteurs naturels et anthropiques, et d'utiliser les communautés animales étudiées comme indicateurs écologiques supplémentaires de l'état environnemental.

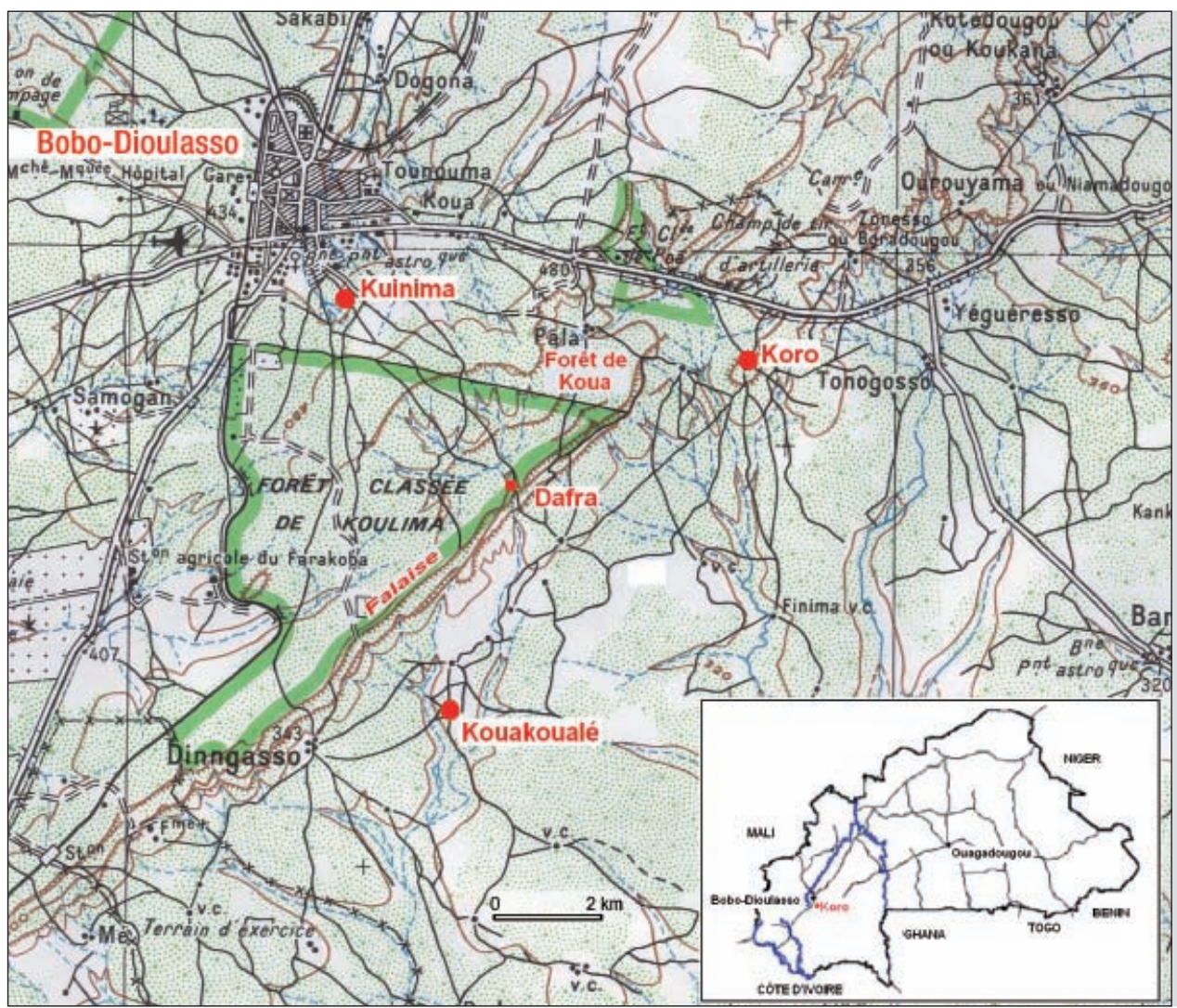

Figure 1.

Carte de situation (d'après carte de l'Institut géographique du Burkina Faso au 1/200 000). 


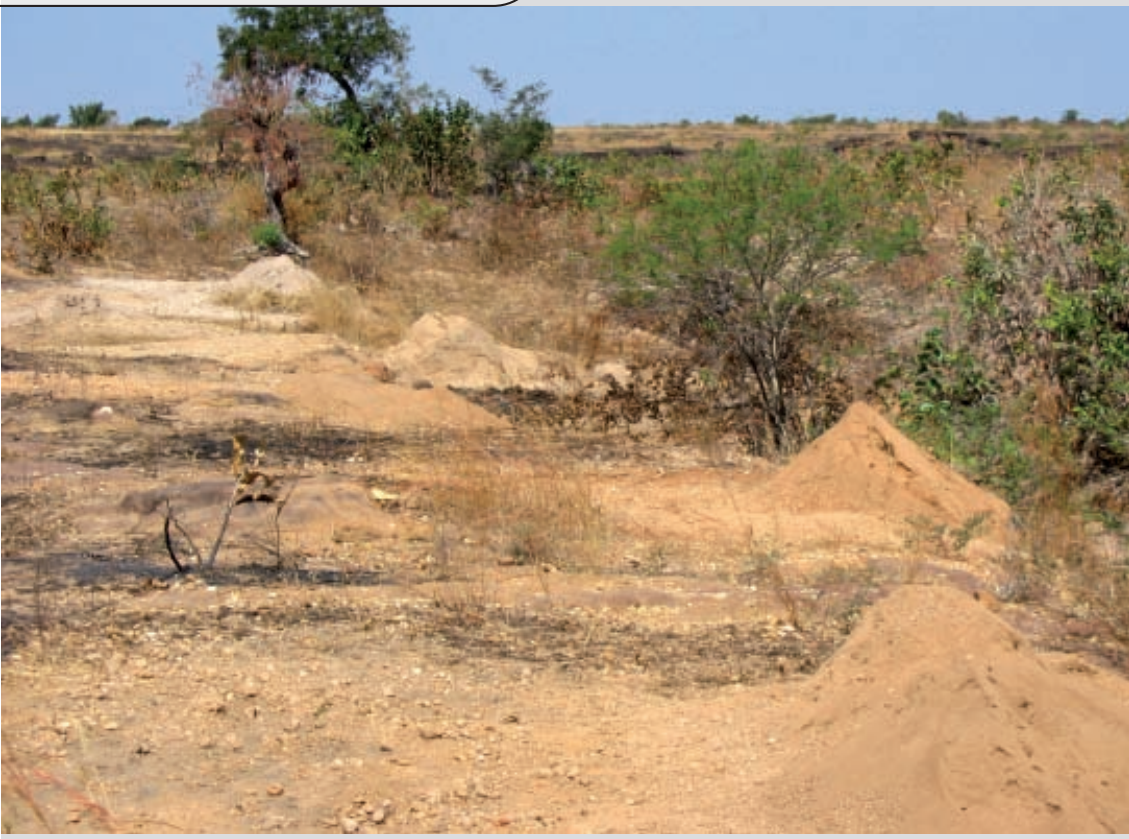

Photo 2.

Exploitation artisanale du sable dans la forêt classée de Koua. Photo J. César.

bilité de l'espèce. Les meilleures espèces reçoivent la note 5 , celles qui ne sont jamais broutées sont notées 0 . La valeur pastorale globale du pâturage est obtenue en multipliant la contribution spécifique de chaque espèce par son indice de valeur pastorale. La valeur pastorale exprimée en pour cent est la somme de tous ces produits divisée par 5 , comme dans la formule (2) suivante :

$V=\Sigma\left(c_{i} \times v_{i}\right) / 5$

Avec $c_{i}$ issue de la formule (1) et $v_{i}$ l'indice spécifique de valeur pastorale, notée de 0 à 5.

Chaque transect a été inventorié en juillet et en octobrenovembre des années 2006 et 2008. L'intervalle de deux ans s'est révélé suffisant pour évaluer

Les formations végétales étudiées sont variées. Elles ont été choisies en fonction de leur caractère relictuel stable, parfois aussi pour leur vulnérabilité (berge de cours d'eau, sites fréquentés). Il s'agit de galeries forestières, de forêts en tête de talweg, autour des sources issues des falaises gréseuses. Ce sont parfois des lambeaux de forêts denses de plateau abritant un cortège d'espèces reliques soudano-guinéennes, voire de forêts claires et de savanes diversement arborées. Treize lignes d'observation (transects) balisées par des repères permanents ont été placées dans les quatre sites. Souvent ces lignes d'observation ont été volontairement placées à la limite entre deux formations pour mieux comprendre l'évolution de l'une vers l'autre.

La méthode d'inventaire utilisée est celle décrite par DAGET et PoIsSONET (1971), qui consiste à effectuer un comptage des espèces herbacées entrant en contact avec un ruban gradué. La contribution spécifique est calculée pour exprimer le pourcentage de chaque espèce dans la végétation avec la formule (1) suivante :

$c_{i}=$ (nombre de contacts de l'espèce $i /$ nombre total de contacts) $\times 100$.

L'échantillonnage des strates ligneuses est obtenu par une méthode dérivée qui permet également de déterminer la structure verticale de la végétation (CÉSAR, ZOUMANA, 1999). Le long d'un transect de 50 mètres $(\mathrm{m})$, un comptage est effectué tous les 50 centimètres $(\mathrm{cm})$ et tous les mètres en hauteur grâce à une perche télescopique de $9 \mathrm{~m}$ de haut.

La valeur pastorale des sites herbacés pâturés a été calculée (DELPECH, 1960). Elle permet d'évaluer la valeur globale d'un herbage à partir d'un relevé en contribution spécifique. À chaque espèce est attribué un indice spécifique de valeur pastorale, notée de 0 à 5 . Cet indice tient compte à la fois de l'appétibilité, de la valeur alimentaire et de l'accessi- les vitesses de croissance des ligneux et observer les diverses perturbations : pâturage sélectif, broutage de ligneux, coupe d'arbres, dégradation du couvert végétal.

Les bioindicateurs animaux ont été inventoriés sur les mêmes sites que la végétation. Il s'agit des insectes (cétoines et papillons frugivores à l'état adulte), des rongeurs et des chauves-souris. Ces groupes ont en effet déjà montré leur utilité dans l'évaluation des conséquences des changements globaux sur l'environnement (MEYER et al., 2010 pour les chauves-souris ; BOUYER et al., 2007 pour les insectes; DUPLANTIER, 1998 pour les rongeurs).

Les insectes bioindicateurs ont été capturés à l'aide de pièges à appât alimentaire (bananes fermentées) selon une méthode décrite par AKoudjın et al. (2011). Les inventaires entomologiques réalisés dans le parc tri-national du W et dans la forêt du Kou à Dindéresso, une forêt-galerie particulièrement bien conservée par la mise en réserve pour la protection des sources d'eau potable, ont servi de références et de comparaisons pour quantifier les dégradations biologiques et environnementales des savanes boisées et des forêts des sites d'étude.

Les rongeurs et les chauves-souris ont été échantillonnés et identifiés selon les méthodes et critères classiquement utilisés dans ces groupes en Afrique de l'Ouest (FaHr, sous presse ; GRANJon et al., 2004 ; GRANJon, Duplantier, 2009 ; KALKO, FAHR, sous presse). Les résultats ont été comparés avec ceux obtenus dans des sites forestiers voisins du Mali (région de Sikasso) et du Burkina Faso (Granjon, DUPLANTIER, 2009 ; KANGOYÉ et al., en préparation).

Le diagnostic écologique effectué ensuite s'appuie sur l'ensemble de ces observations, inventaires des sites et études écologiques. Il a débouché sur la constatation de graves dégradations dans les trois zones étudiées : le plateau au-dessus de la falaise, la falaise elle-même et les plaines au pied de la falaise. 
Les propositions d'amélioration, les remèdes

Des expérimentations sur le terrain ont été menées directement chez des producteurs. Ces expérimentations incluent des dispositifs antiérosifs, des essais fourragers et des plantations de ligneux d'espèces locales ou introduites. Des essais agronomiques classiques ont aussi été installés en station, en particulier pour les améliorations fourragères (CÉSAR et al., 2009). L'ensemble de ces essais avait pour but de tester les conditions de sol, souvent limites, et l'acceptabilité des techniques par les populations d'agriculteurs et d'éleveurs. Les résultats détaillés peuvent être trouvés dans le rapport final du projet Fsp (CIRDES, 2009), servant de référence à cette étude pour proposer des solutions à la dégradation observée.

\section{Le constat écologique}

Le complexe des falaises gréseuses de Banfora comprend, dans la zone de Bobo-Dioulasso, trois systèmes écologiques bien distincts :

- le plateau au-dessus de la falaise, sur lequel est installée la ville de Bobo-Dioulasso ;

- la falaise proprement dite, qui s’étage en plusieurs escarpements de roches gréseuses, séparés par autant de replats occupés par les savanes et forêts claires ; - la plaine et le pied de la falaise.

Le plateau est essentiellement le domaine d'une agriculture intensive et d'activités de cueillette liées à la présence de la ville.

La falaise, peu apte à l'agriculture, abrite des sites cultuels dont certains sont aussi devenus touristiques.

La plaine au pied de la falaise est le domaine de l'agriculture intensive car elle bénéficie de conditions hydriques privilégiées. Ailleurs dans la plaine, l'agriculture est extensive. L'élevage y est abondant.

\section{Le plateau}

\section{La dégradation des galeries forestières}

Une dégradation généralisée des galeries forestières dans les environs de Bobo-Dioulasso a été observée (BouYER et al., 2005 ; GUERRINI et al., 2008). La végétation ligneuse ripicole y est exploitée pour le bois ou éliminée pour l'installation de cultures. Les habitants du village de Kuinima, installé au-dessus de la falaise, ont depuis longtemps déboisé le plateau y compris les galeries forestières pour l'exploitation du bois et les défrichements culturaux. Les quelques grands arbres forestiers isolés qui restent témoignent du passé. Les champs s'étendent jusqu'aux berges dénudées des cours d'eau.
Les captures réalisées à l'emplacement de ces anciennes galeries forestières montrent que la faune entomologique forestière s'est fortement réduite. Le nombre d'espèces forestières recensées est bien moindre que dans la forêt du Kou (Dindéresso), qui est la référence de cette étude. La persistance de certaines espèces rares témoigne cependant de leur ancienne richesse faunique et floristique. Ainsi, aucune des six espèces de papillons forestiers de la forêt du Kou n'a été retrouvée (dont Charaxes tiridates Cramer, C. brutus Cramer, C. boueti Feisthamel, Bicyclus sandace Hewitson). Par contre, seulement trois des six espèces de cétoines forestières du Kou ont pu être relevées (Chlorocara africana Drury, C. guerrini Janson et Eudicella daphnis Buquet) à des densités significativement inférieures. À titre d'exemple, un seul spécimen de Chlorocara africana a été piégé à Dafra en trois campagnes de 10 jours de piégeage avec cinq pièges, alors qu'à Toussiana, dans la forêt relicte de la falaise distante de $50 \mathrm{~km}$ environ de BoboDioulasso, 14 spécimens ont été piégés en cinq jours avec cinq pièges couvrant tout juste le quart de la superficie de cette forêt et 48 individus ont été capturés dans la forêt du Kou avec 17 pièges en 10 jours.

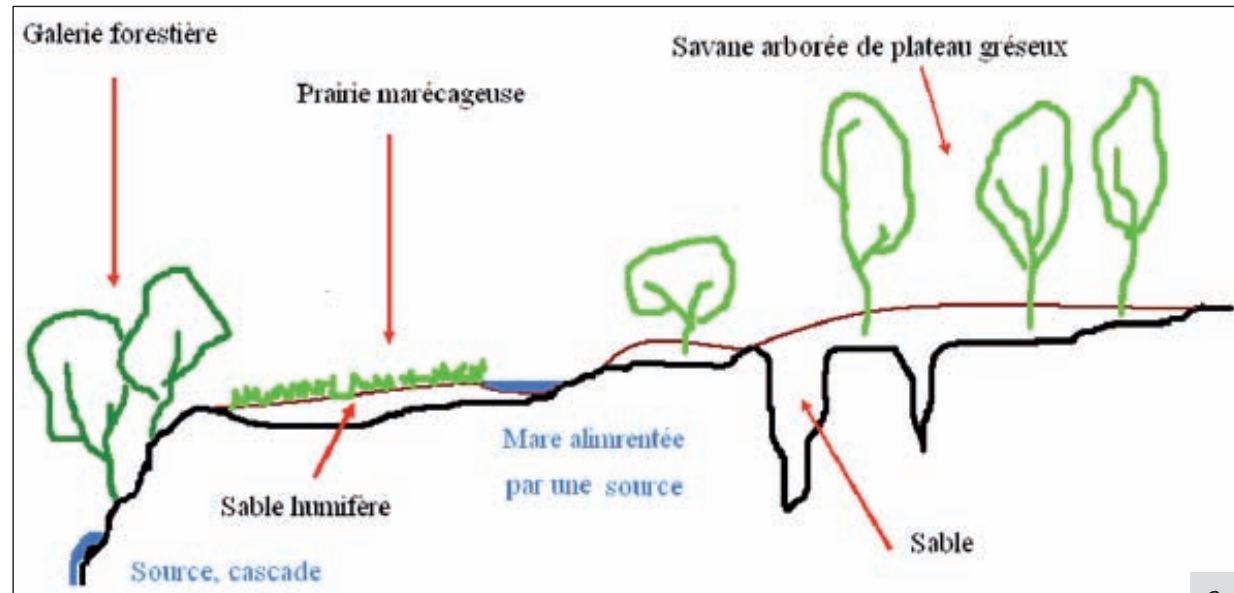

Galerie forestiere

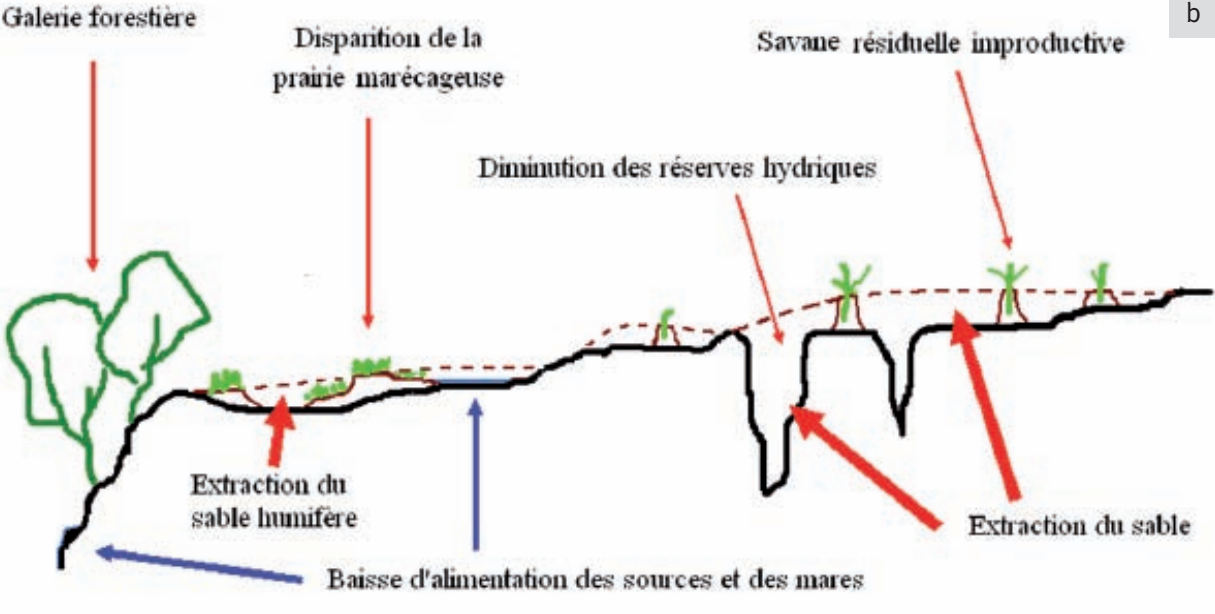

Figure 2.

Le rebord du plateau au niveau de la source de Dahana

à Koro ; (a) état originel ; (b) état actuel. 
De la même façon, la diversité des rongeurs des sites de Kuinima apparaît particulièrement faible, avec rarement plus de trois espèces rencontrées (voir la figure 7 dans CÉSAR et al., 2010). Les communautés présentes sont dominées par des espèces généralistes voire anthropophiles (Mastomys erythroleucus, Rattus rattus), tandis que les espèces typiquement forestières à affinités biogéographiques soudano-guinéennes (voir la liste dans GRANJON, DUPLANTIER, 2009) sont absentes de ces sites. Une telle régression faunique confirme l'inquiétante dégradation anthropique des galeries forestières.

\section{La dégradation des savanes et des forêts claires}

Si l'on prend l'exemple de la forêt de Koua à l'Ouest de Koro, qui occupe le plateau au-dessus de la falaise, cette ancienne forêt claire à Terminalia laxiflora Engl. (Combretaceae) avait été classée par l'administration forestière afin de préserver les sources du pied de la falaise. Actuellement, elle est encore protégée par un projet du $7^{\text {e }}$ Fonds européen de développement (FED, 7 ACP BK /031) qui ne parvient pas à stopper l'exploitation intensive du bois (photo 1). La forêt claire a aujourd'hui disparu pour faire place à un taillis lâche. Les rejets de souche sont coupés dès qu'ils atteignent 3 ou $4 \mathrm{~cm}$ de diamètre. Seules subsistent d'anciennes plantations de Gmelina arborea Roxb. (Verbenaceae) réalisées en 1978 par les villageois pour protéger les sources d'eau, plantations dont les villageois ont ensuite affirmé qu'elles en avaient amélioré le débit.

L'exploitation du sol pour le sable et les graviers comme matériaux de construction (photo 2) a des conséquences environnementales qui nous semblent encore plus graves. Les crevasses entre les blocs de grès du plateau sont vidées de leur sable qui est raclé jusqu'à la roche. Le sable est aussi prélevé sur des lentilles de faible profondeur, ce qui augmente notablement l'ampleur des surfaces où les sols sont détruits. Cette pratique entraîne l'impossibilité de cultiver ou de régénérer la végétation forestière. Elle a aussi comme conséquences une diminution des réserves hydriques et des perturbations du réseau d'écoulement des eaux de surface (figure 2). L'absence de stockage temporaire des eaux de pluie dans des sols inexistants induit un accroissement de la violence des crues et une érosion importante des berges, mis en évidence par les observations faites sur une période de deux ans pendant laquelle $10,75 \mathrm{~m}$ de berges sableuses ont été emportés (figure 3).

Une diminution de la densité apparente des lépidoptères (ex. Hamanumida daeadalus Fabricius, Charaxes epijasius Reiche) (photos 3 et 4) et de la diversité du cortège des insectes de savane a été mesurée. À des densités significativement plus faibles, les cinq espèces de papillons de savane rencontrées dans le parc du W ont été retrouvées. Par contre, seulement 11 des 16 espèces de cétoines savanicoles de ce parc (BOUYER et al., 2007 ; AKOUdנIN, 2011 ; AKOudנIN et al., sous presse) ont pu être collectées. L'effort de capture des rongeurs dans la forêt de Kou a été faible, mais une seule espèce généraliste à tendance anthropophile (Cricetomys gambianus) y a été capturée. Ces modifications de la faune sont le reflet de la dégradation importante de ces milieux savanicoles en bordure de la falaise.
Figure 3.

Matérialisation d'une ligne d'observation mise en place en 2006. Entre 2006 et 2008, entourés sur la photo, 10,75 m de berge sableuse ont été emportés par les crues violentes. 

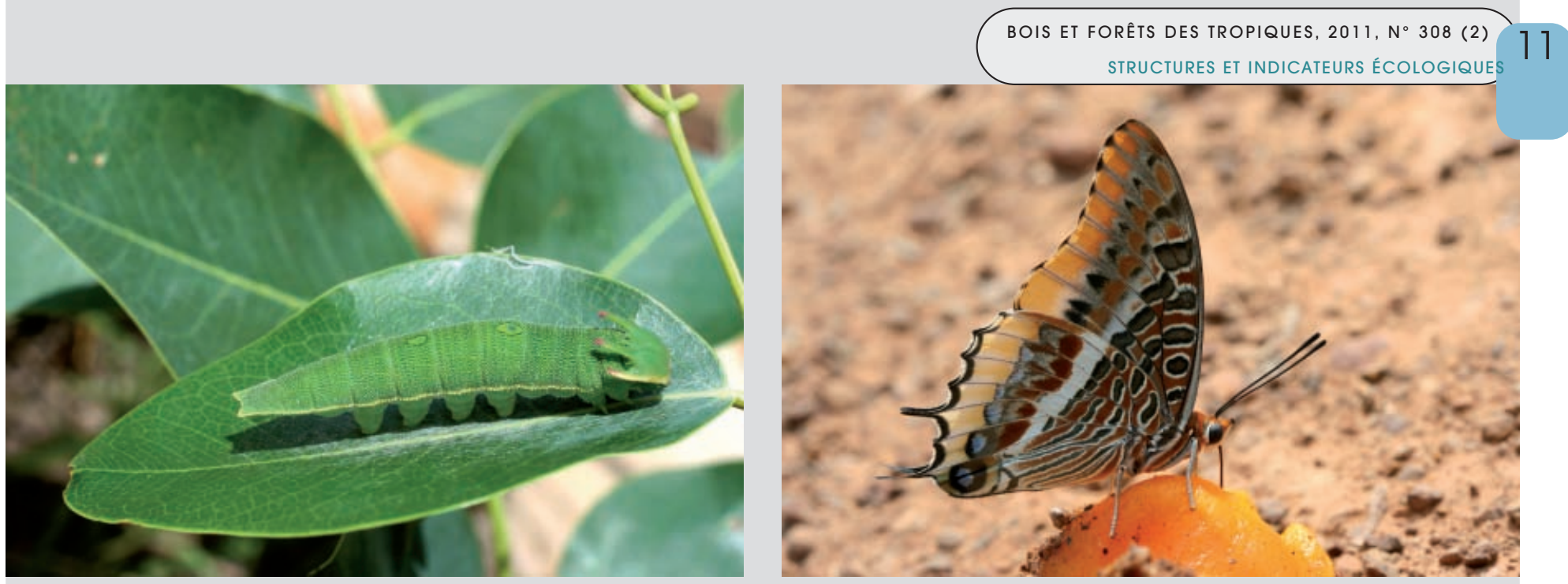

Photos 3 et 4 .

Charaxes epijasius, papillon de savane : larve sur Afzelia africana et adulte se nourrissant sur une mangue, falaise de Banfora.

Photos J. Bouyer.

\section{La falaise}

\section{L'exploitation pastorale}

Du plateau à la plaine en contrebas, de nombreux replats couverts de savane sont accessibles au bétail. Le relief en rend toutefois l'accès difficile. Le bétail y circule, entraînant des dégradations minimes.

\section{L'exploitation du bois}

Les défrichements pour l'approvisionnement en bois de la ville de Bobo-Dioulasso n'épargnent pas la falaise. Les grands arbres ont disparu depuis longtemps près de BoboDioulasso. Des femmes âgées coupent les rejets de souche dans les zones accessibles de la falaise. À Koro et Kouakoualé, les gros arbres sont aussi exploités sur la plaine en contrebas. Ce n'est que vers Toussiana, à près de $50 \mathrm{~km}$ au sud, que se rencontrent les premières forêts claires à Isoberlinia doka Craib \& Stapf et Isoberlinia tomentosa (Harms) Craib \& Stapf (Caesalpiniaceae). Ces forêts encore naturelles risquent cependant de disparaître rapidement car les charbonniers s'y installent actuellement.

\section{Les sites sacrés}

Il existe de nombreux sites de sacrifices rituels dans la falaise. Ces sites sacrés ne sont pas épargnés. Un lieu aussi important sur le plan cultuel que Dafra, où des sacrifices rituels ont lieu journellement, voit néanmoins sa relicte de forêt se réduire chaque jour. Le microclimat de cette galerie forestière encaissée dans une gorge rocheuse est perturbé par la disparition de la forêt claire sur le plateau supérieur (photo 5). De grands arbres, Berlinia grandiflora (Vahl) Hutch. \& Dalziel (Caesalpiniaceae), Lannea acida A. Rich. (Anacardiaceae), sont abattus à proximité du site sacré (photo 6). Dans le bas de la falaise, des jardins potagers sont installés après abattage de la végétation ripicole (photo 7).

La disparition de la forêt claire entraîne progressivement la raréfaction des espèces animales forestières. Les espèces qui persistent comme les cétoines, Chlorochara africana et Eudicella daphnis (photos 8 et 9), pourraient être menacées

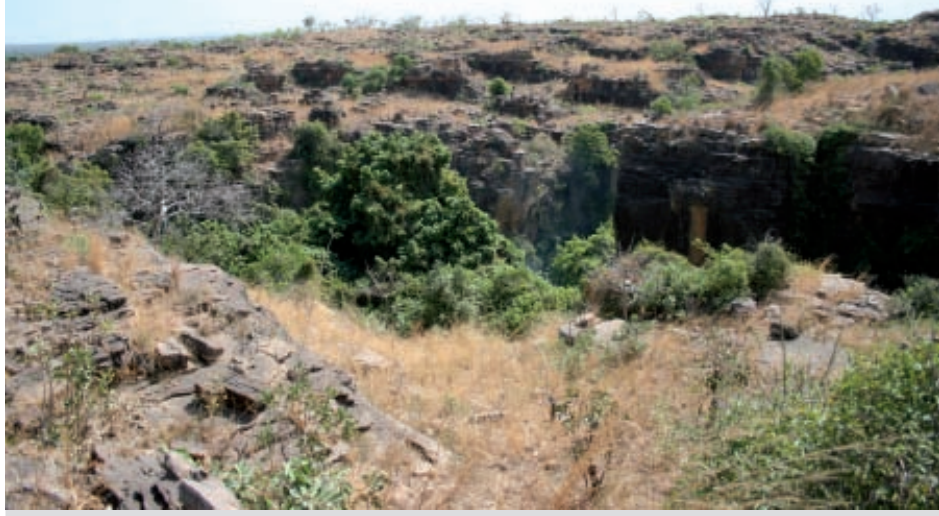

Photo 5.

Le plateau dénudé au-dessus de Dafra perturbe le microclimat et fragilise la forêt.

Photo J. Bouyer.

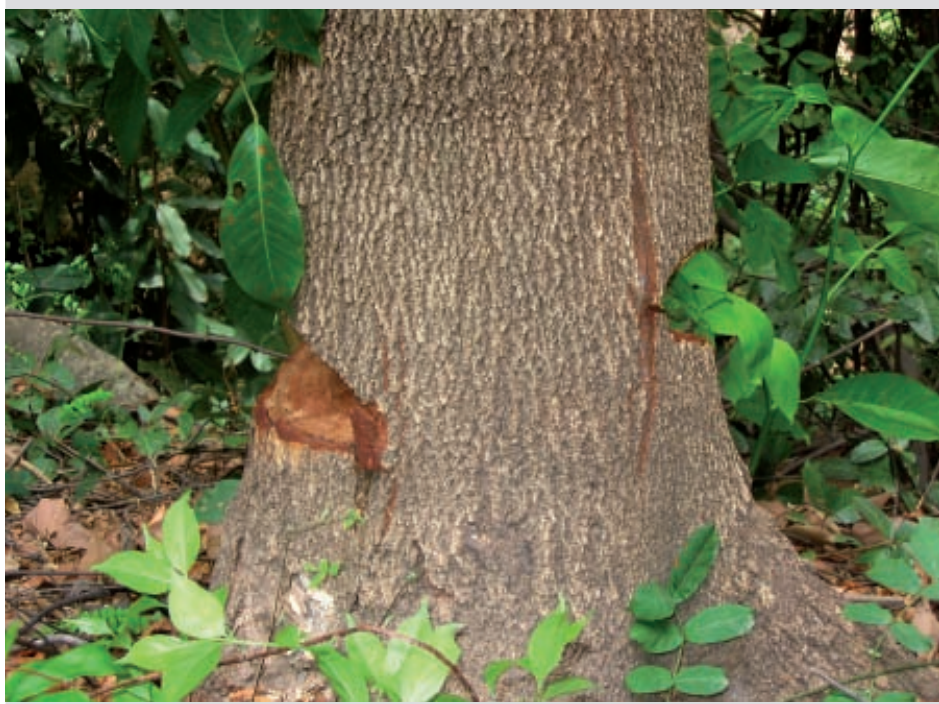

Photo 6.

Un Berlinia grandiflora convoité par un bûcheron

à proximité du lac de Dafra.

Photo J. César. 


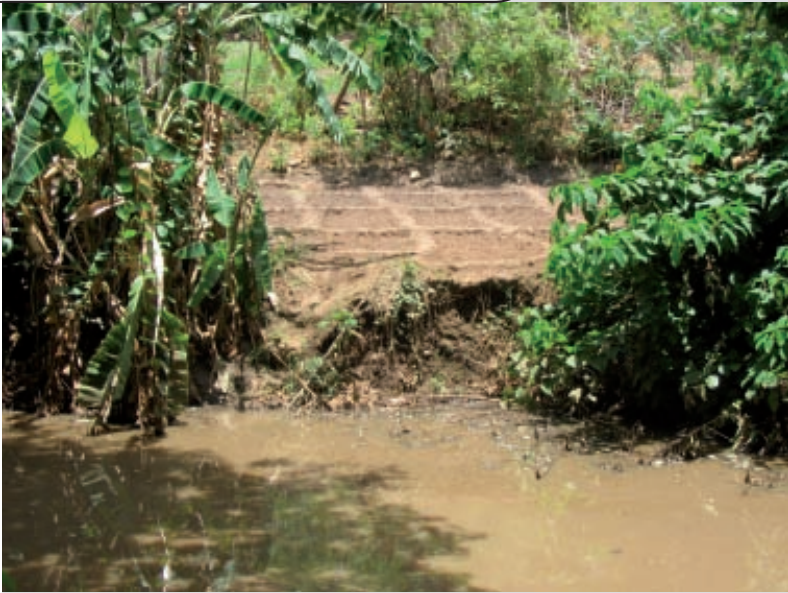

Photo 7.

Jardin potager dans la galerie forestière de Dafra provoquant la destruction de la forêt et l'effondrement des berges. Photo J. César.
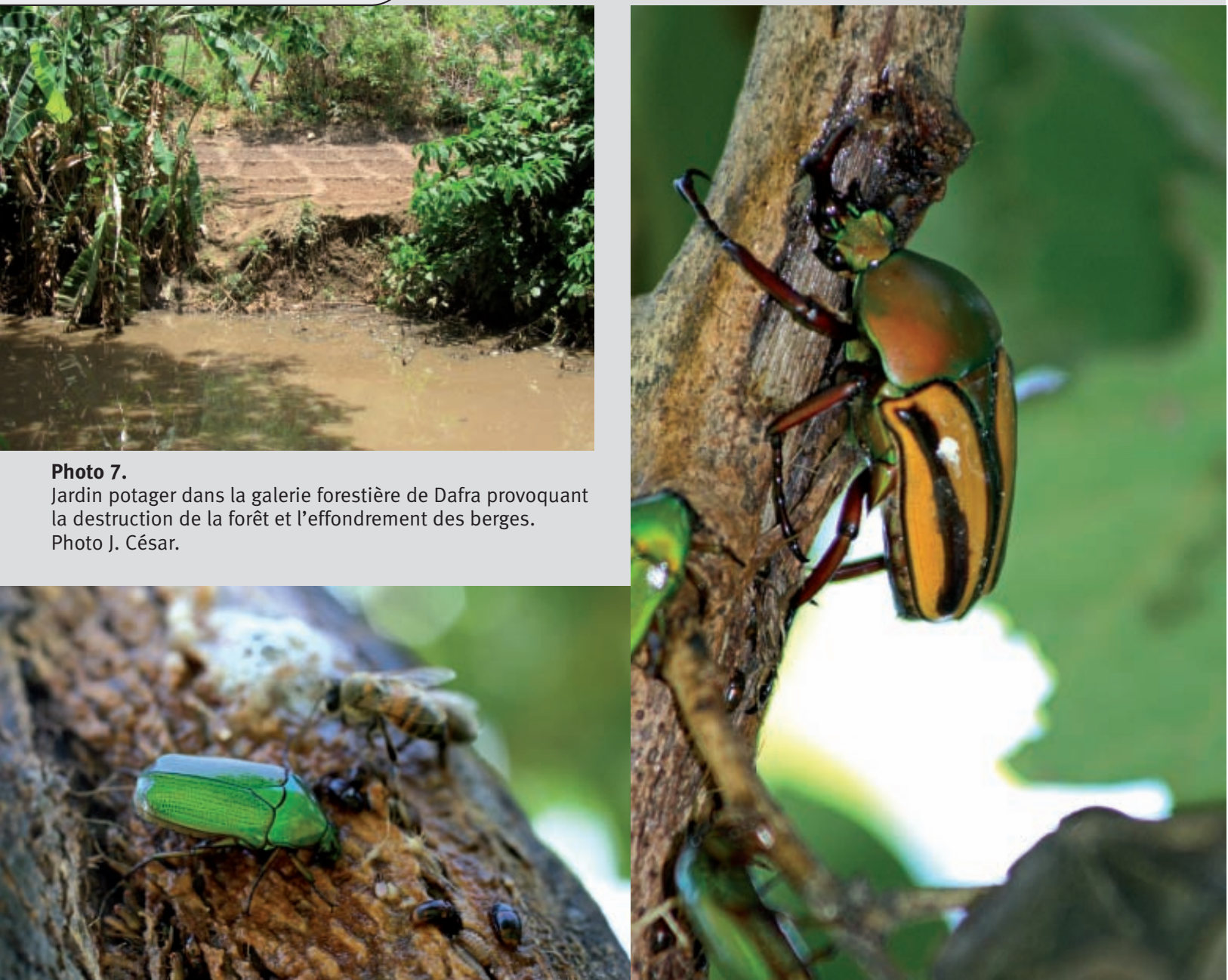

Photos 8 et 9.

Chlorochara africana et Eudicella daphnis, cétoines forestières en cours de disparition, s'alimentant sur les exsudats en fermentation d'un Cordia mixa à proximité du site de Dafra.

Photos J. Bouyer.

de disparition comme c'est le cas sur le plateau, car elles ne se rencontrent plus que dans deux des cinq sites inventoriés (CÉSAR et al., 2010). Le ramassage du bois mort accélère la régression de ces espèces dont les larves sont détritivores. La pénétration dans la galerie forestière de Dafra du rat noir (Rattus rattus Linnaeus, photo 10), espèce commensale invasive, à partir des jardins en aval (photo 7), témoigne de la dégradation de ce milieu. Le phénomène est encore plus flagrant dans la petite forêt-galerie voisine, à l'aval de la cascade de Kou située près du village de Koro, autour des bassins à silures géants. Là, le rat noir est la seule espèce de rongeur rencontrée alors qu'elle coexiste avec une espèce locale ubiquiste à Dafra, Mastomys erythroleucus Temminck. Des petits rongeurs partiellement arboricoles et à tendance forestière, comme Praomys daltoni Thomas (photo 11) et Praomys rostratus Miller (voir la photo 2 dans CÉSAR et al., 2010), ne se rencontrent plus dans ces fragments forestiers dégradés. En revanche, une communauté de chauves-souris relativement diversifiée - incluant en particulier les espèces forestières Hipposideros jonesi Hayman, Nycteris hispida Schreber et, dans une moindre mesure, Neoromicia guineensis Bocage - semble s'y maintenir.
L'exploitation commerciale d'espèces ligneuses recherchées pour leur bois, Bombax costatum Pellegr. \& Vuillet (Bombacaceae), Pterocarpus erinaceus Poir. (Fabaceae), Berlinia grandiflora et d'autres, entraîne à Dafra et dans d'autres sites sacrés de la falaise une raréfaction des grands arbres et de la faune qu'ils abritent. Leur remplacement progressif par des espèces commensales devient déjà localement dominant.

\section{L'exploitation du sable}

Le sable pour la construction est aussi exploité dans la falaise (photo 12), notamment sur les replats accessibles aux camions. À tous les niveaux de la falaise, le sol est creusé et tamisé pour séparer le sable des graviers. Les éboulis stabilisés du pied de la falaise qui portent une savane arborée à Terminalia laxiflora sont riches en graviers. Ils sont transformés en carrière partout où ils sont accessibles. Or, ces éboulis sont des zones où les sols sont profonds, bien que rocailleux. L'eau de pluie, qui a ruisselé des plateaux gréseux où elle ne peut s'infiltrer, pénètre aisément dans ces sols meubles. L'infiltration peut y être localement estimée au double des précipitations. Ces 


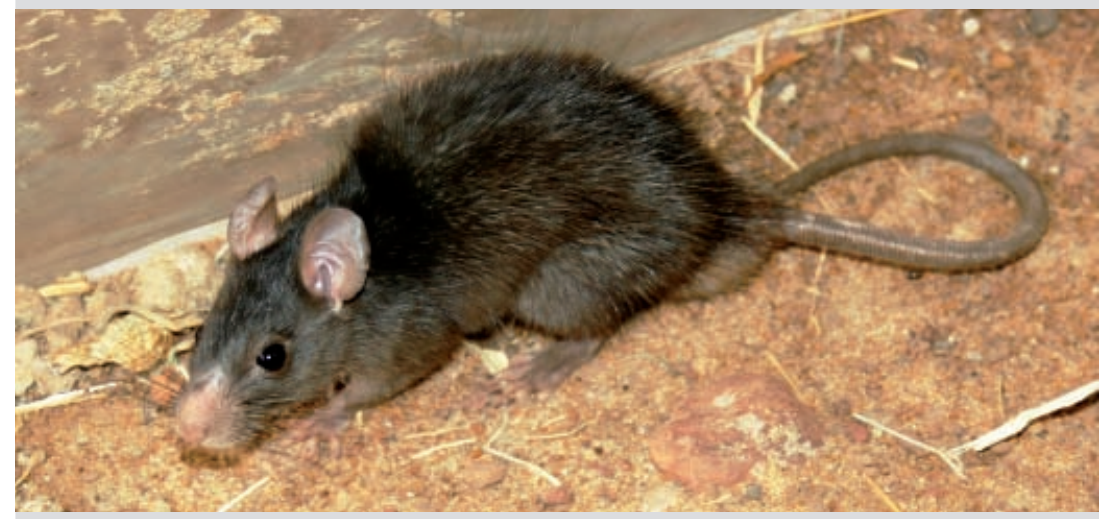

Photo 10.

Rattus rattus.

Photo L. Granjon.

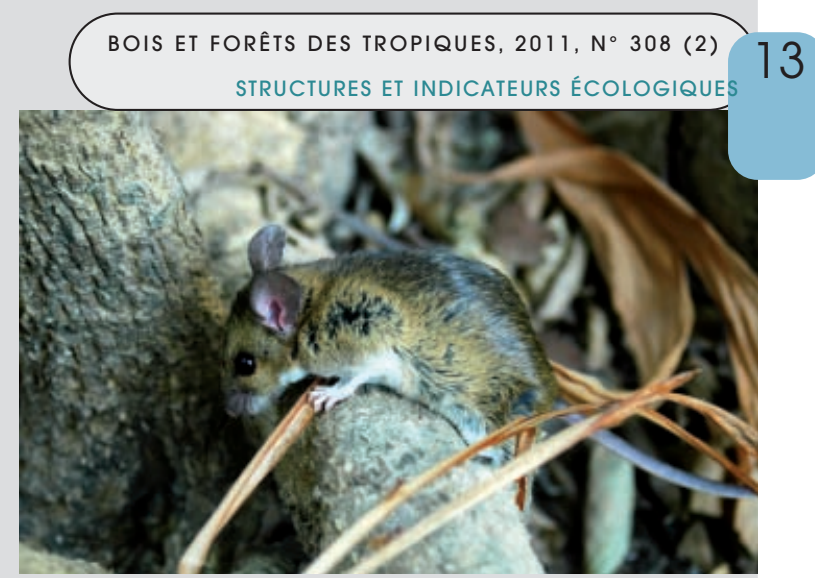

Photo 11.

Praomys daltoni.

Photo L. Granjon. conditions permettent à des espèces exigeantes en eau de pousser (voir les figures 1 et 2 dans CÉSAR et al., 2010). La végétation y est dense, dynamique et sa croissance est rapide. Les arbres peuvent y atteindre des dimensions importantes que l'on ne rencontre pas dans la plaine ou sur le plateau, sauf dans les forêts-galeries. En détruisant les forêts de piémont, les carriers perturbent le régime hydrique du milieu, réduisent l'alimentation des nappes phréatiques, accélèrent le tarissement précoce des sources du pied de la falaise en saison sèche et accroissent l'importance des crues occasionnelles.

\section{La plaine et les savanes au pied de la falaise}

\section{La destruction des galeries forestières}

Les galeries en aval de la falaise sont aussi menacées par le maraîchage qui s'installe le long des cours d'eau. Considérés comme gênants pour les jardins, les ligneux sont abattus. Des galeries forestières ont ainsi été complètement défrichées. Les berges qui n'étaient pas maintenues par les racines des arbres se sont progressivement effondrées (photo 13).

La dégradation des galeries forestières, des berges des ruisseaux et des savanes attenantes, du fait des cultures et du surpâturage, a des conséquences sur la faune de ces milieux, notamment l'homogénéisation des populations de rongeurs, avec l'apparition d'une surdominance d'espèces généralistes et ubiquistes, en particulier Mastomys erythroleucus (photo 14).

\section{La pression pastorale}

Le pâturage entraîne souvent de graves dégradations de la végétation dans la plaine. Dans la réserve sylvopastorale de Tontorama sur le territoire de Koro, l'indice de valeur pastorale de la végétation herbacée, qui caractérise la qualité alimentaire de l'herbage, a régressé de 60 à $38 \%$ en deux ans. De vastes surfaces de sol sont compactées par le piétinement du bétail et pourraient être perdues pour l'agriculture sans un investissement important. Le tapis herbacé est devenu inexistant et, lorsque le sol n'est pas nu, les seuls végétaux sont des ligneux indicateurs de la dégradation du milieu, résultant de l'embroussaillement par le surpâturage : Dichrostachys cinerea (L.) Wight \& Arn. (Mimosaceae), Cassia sieberiana DC. (Caesalpiniaceae), Guiera senegalensis J.F. Gmel. (Combretaceae).

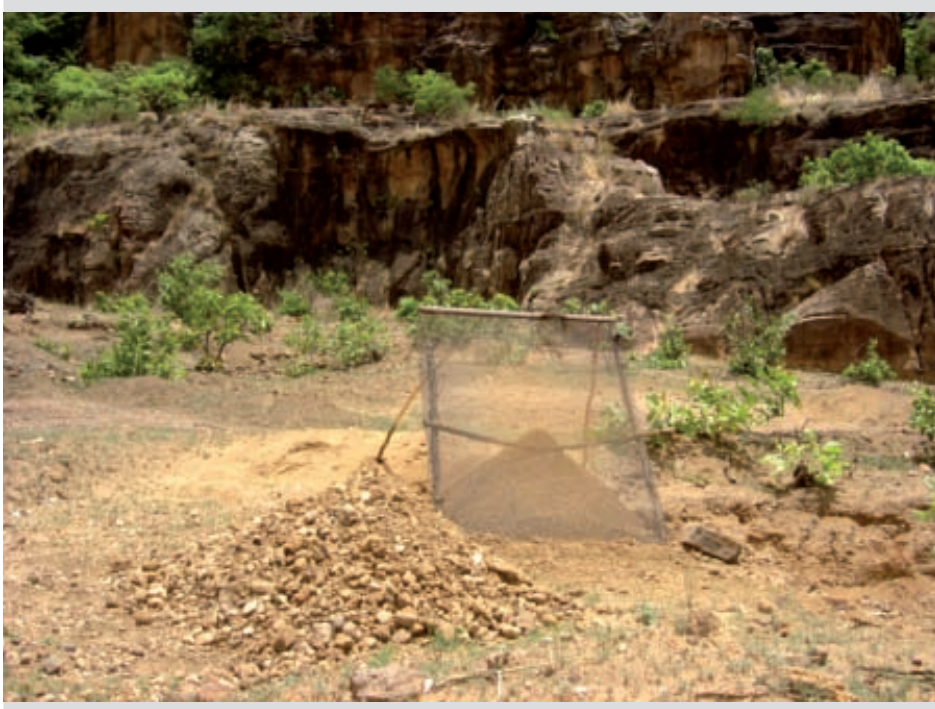

Photo 12.

L'extraction du sable et du gravier sur le piémont de la falaise.

Photo M. Akoudjin.

\section{L’érosion}

Le bas-fond de Koro, traversé par la rivière Kou, issue de la cascade du même nom, était encore vers 1950 couvert d'une végétation dominée par Mitragyna inermis, arbre indicateur de zones humides aux sols lourds, argileux et mal drainés, et peuplé de crocodiles (Osteolaemus tetraspis Cope). Dans les années 1970, la culture maraîchère et les vergers de bananiers s'installent progressivement. Les cultures maraîchères sont irriguées à partir de petits canaux. Jusqu'à l'année 2000, le cours d'eau n'avait pas de lit creusé et la plaine d'épandage était entièrement inondée en saison des pluies. En 2002, une carrière de sable est creusée en aval de la plaine. C'est le début d'une érosion régressive : un lit se creuse et atteint rapidement la roche mère (photo 15). Des griffes d'érosion entament les berges. Celles-ci ne sont pas protégées mais labourées par les agriculteurs jusqu'à leurs bords extrêmes et s'éboulent. La surface cultivable et les revenus diminuent (photo 16) pour avoir voulu cultiver quelques pieds de maïs de plus. Les terres maraîchères de Koro sont ainsi menacées. Les autorités locales sont impuissantes pour s'opposer aux intérêts individuels. 


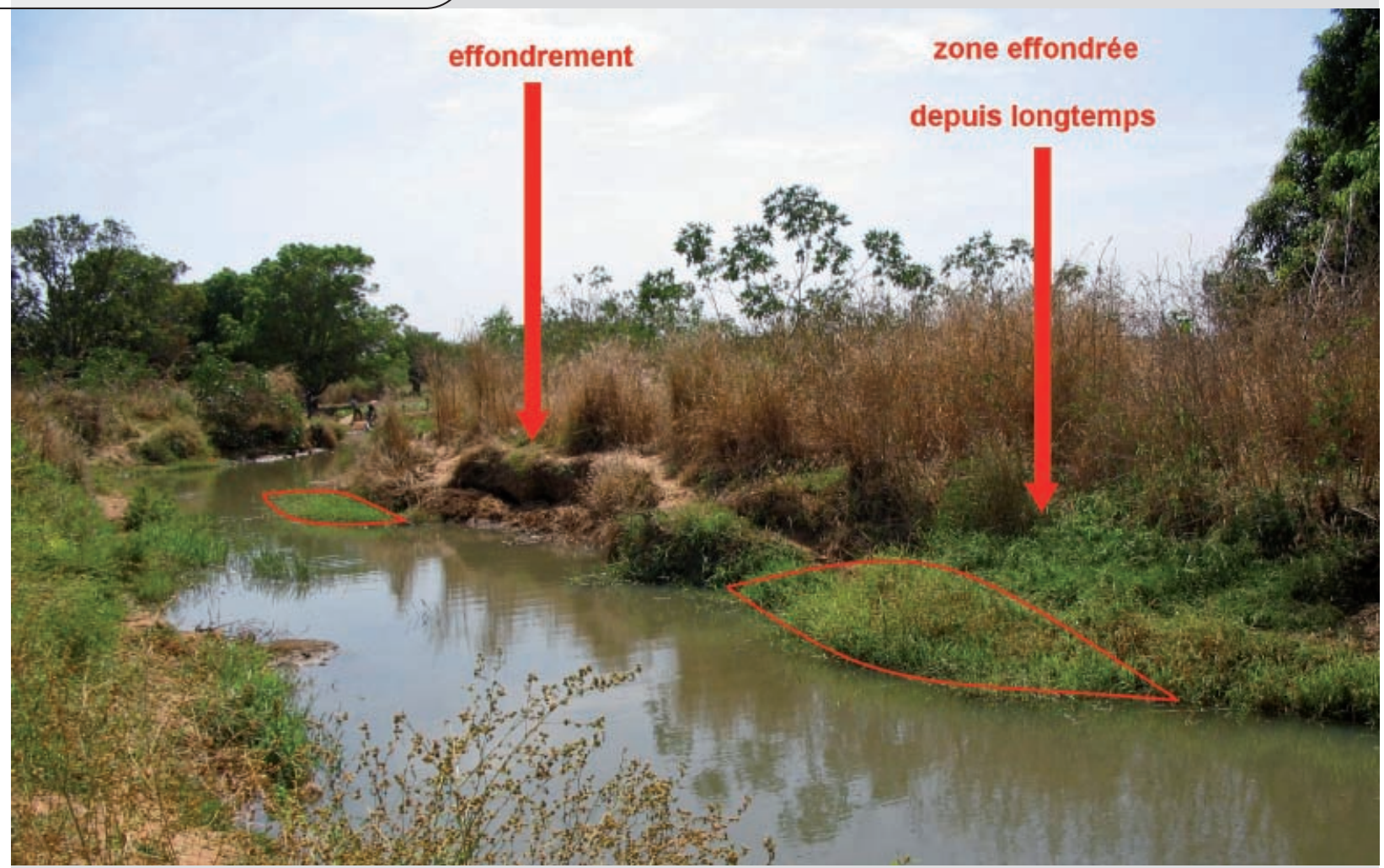

Photo 13.

Dégradation des berges par les défrichements.

Photo J. César.

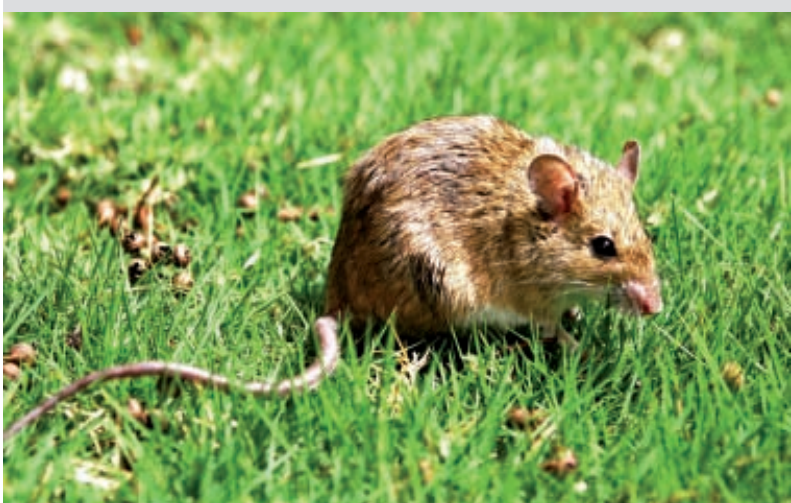

Photo 14.

Mastomys eythroleucus.

Photo J.-J. Lemasson.

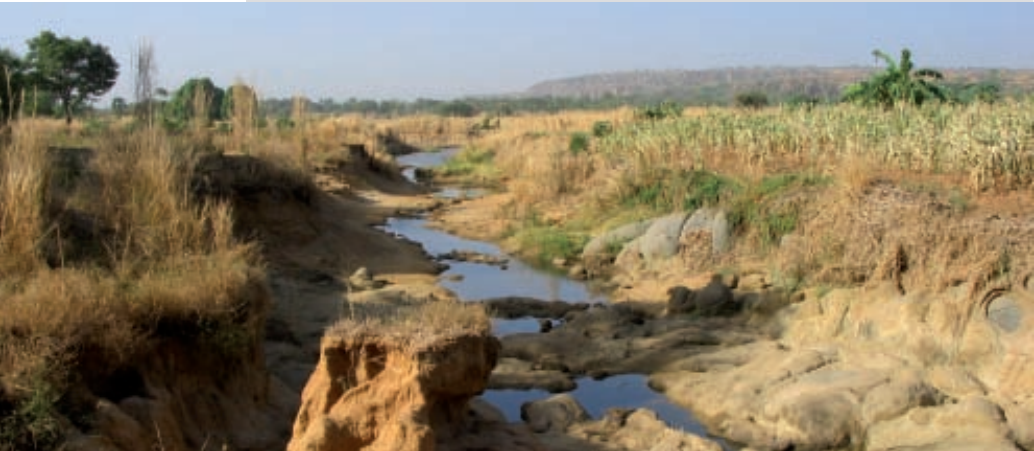

Photo 15.

La plaine de Koro en 2008, récemment tranchée par le lit du Kou.

Photo J. César.
De mauvaises pratiques agricoles aggravent l'érosion. Le labour qui traverse les lignes d'écoulement des eaux sans les éviter, en relevant la charrue, favorise la création des rigoles d'érosion qui s'approfondissent à chaque pluie, provoquent des nappes d'épandage et entraînent des baisses de fertilité importantes (photo 17).

Partout, on constate que ces phénomènes d'érosion prennent de l'importance. Les crues deviennent plus violentes, surtout en début de saison des pluies (DE LA ROCQUE et al., 2004). L'appauvrissement de la végétation naturelle contribue à diminuer l'infiltration des eaux de pluie et leur rétention dans le complexe sol-végétation. Le ruissellement augmente, dans la plaine les méandres s'accentuent et l'érosion s'accroît à une vitesse considérable, jusqu'à emporter des arbres, comme cela se constate avec les photos prises entre 2005 et 2007 à l'entrée de la forêt du Kou à Dindéresso (photos 18).

\section{Conclusion sur les dégradations}

Les dégradations causées par la proximité de la ville de Bobo-Dioulasso dépassent le cadre de la falaise : elles s'étendent sur le plateau et dans la plaine en contrebas. Elles ne sont pas uniquement le fait de l'extension agricole ou de l'exploitation des arbres pour le bois de chauffe. Elles sont aussi la conséquence du développement urbain qui, pour fournir les matériaux de construction, détruit de façon irréversible de grandes surfaces de sol. Les arbres coupés peuvent repousser mais la suppression du sol est irréversible. Cette exploitation minière est de loin la plus grave. 


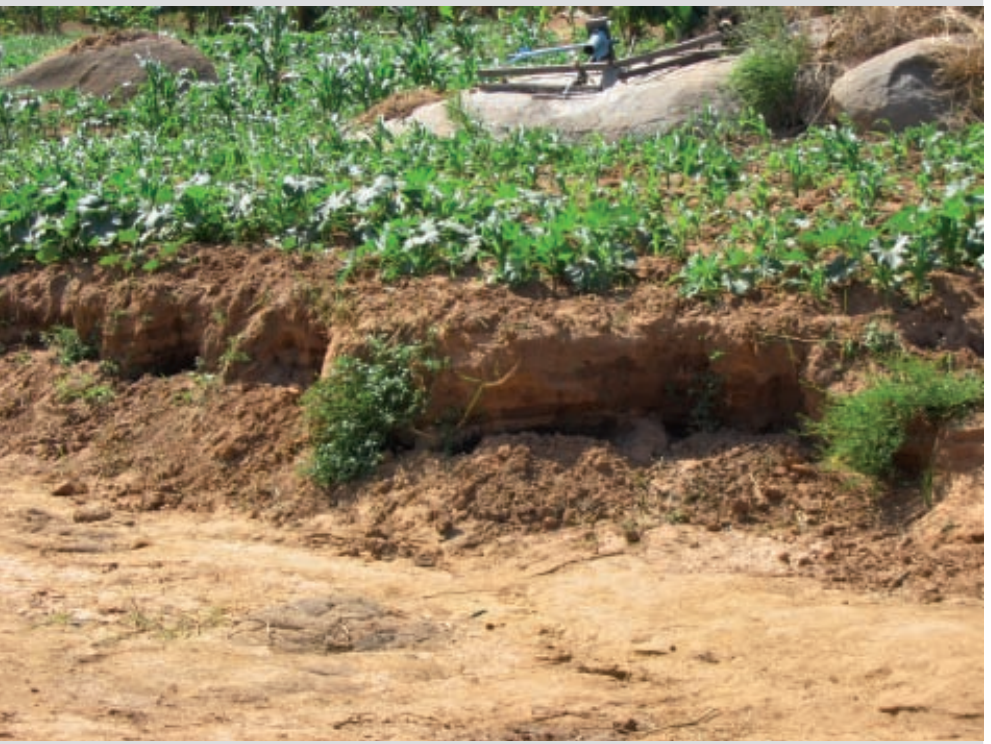

Photo 16.

Champ cultivé trop près de la berge, le sol s'éboule.

Photo J. César.

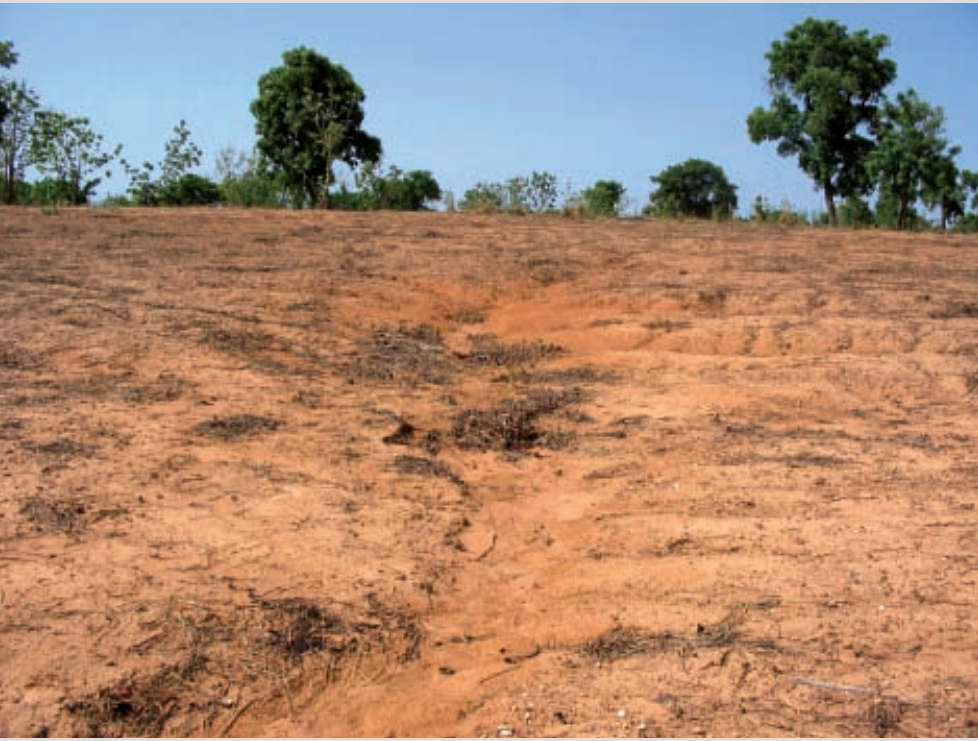

Photo 17.

Rigole d'érosion dans un champ à Koro.

Photo J. César.

\section{Les remèdes}

La lutte contre les dégradations, pour être durable, doit recevoir l'adhésion des populations et les mesures à prendre doivent être en grande partie à leur avantage.

Le projet Fsp, par des améliorations en agronomie, par la diffusion de plantes performantes et de techniques adaptées, avait aussi pour objectif de rehausser le niveau de vie des habitants.

En luttant contre les dégradations par l'amélioration des pratiques agricoles et d'élevage, il devait être plus facile de les aider à protéger leurs ressources naturelles. C'est la voie qui a été privilégiée.

\section{Les pratiques pastorales}

Ce n'est pas tant le pâturage en soi que les mauvaises pratiques pastorales qui entraînent les dégradations qui s'accompagnent d'une baisse générale de la qualité des herbages. Dans les zones périphériques du parc du W où les éleveurs paient, pour chaque tête de bétail, un forfait annuel de pâturage, les charges pastorales restent faibles et permettent un maintien, voire une augmentation, de la biodiversité animale et végétale sauvage (Bouyer et al., 2007). Il est donc possible d'appliquer des systèmes d'exploitation qui permettent une gestion durable des ressources pastorales optimisant la production animale.

Les feux de brousse sont bien souvent imputés aux pasteurs. Traditionnellement, les éleveurs avaient l'habitude de mettre le feu aux pâturages de graminées vivaces pour favoriser les repousses et les rendre accessibles au bétail. Aujourd'hui, dans les zones à forte pression pastorale, les graminées vivaces ont pratiquement disparu des parcours (CHeVAlLier, 1994) et les maigres plantes annuelles desséchées sont devenues précieuses pour tous les éleveurs. Le feu doit être proscrit et les réserves végétales herbacées sur pied doivent être protégées tant pour alimenter le bétail en saison sèche que pour toutes les autres utilisations, notamment la couverture des toitures ou la production de fumier.

Le piétinement des troupeaux dégrade les galeries forestières à proximité des points d'abreuvement, mais ces dégradations sont peu importantes et localisées (photo 19). Par contre, ce piétinement empêche la régénération naturelle des arbres par semis. La délimitation de points d'abreuvement peut enrayer ce phénomène.

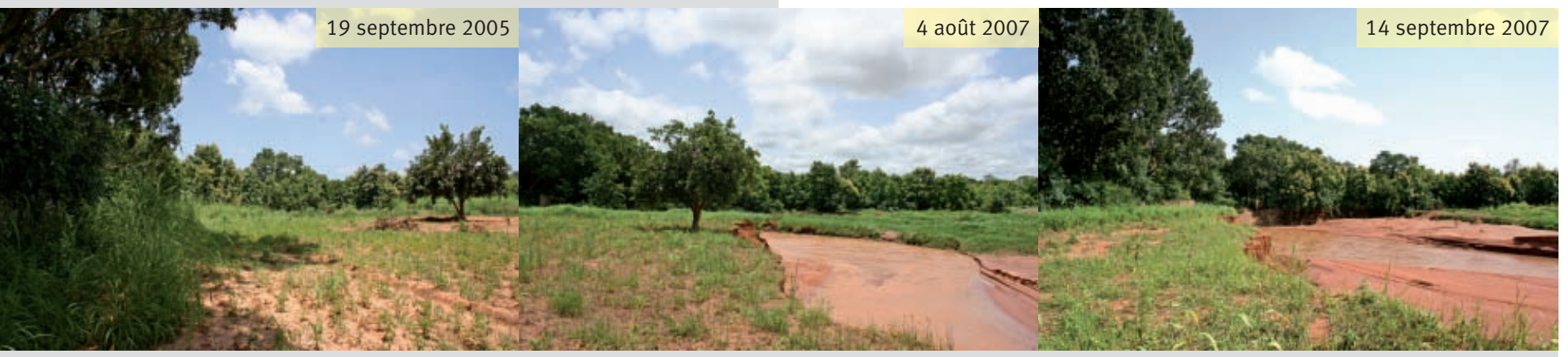

Photos 18.

Effet des crues récentes sur le cours d'une rivière à l'entrée de la forêt classée du Kou.

À gauche, le cours d'eau réduit n'atteint pas l'arbre ; au centre, il s'en approche ;

à droite, l'arbre a été emporté.

Photos J. Bouyer. 


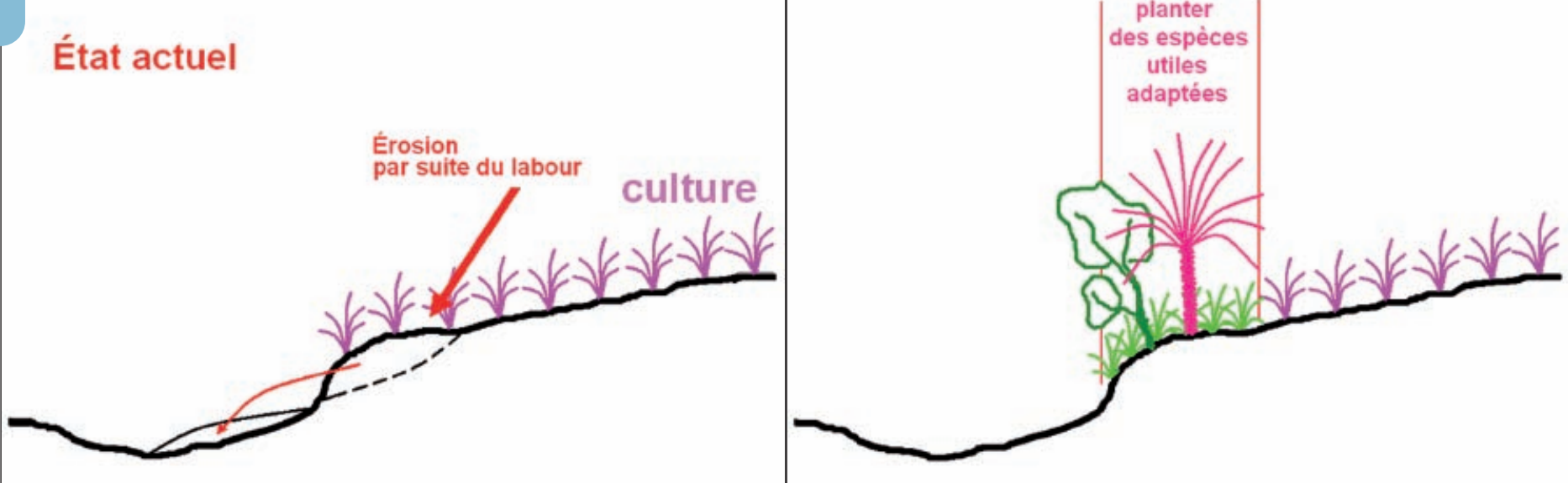

Figure 4.

Le labour jusqu'à la berge réduit la surface du champ. La technique de protection consiste

à laisser l'herbe et les ligneux repousser, et à planter des espèces utiles adaptées.
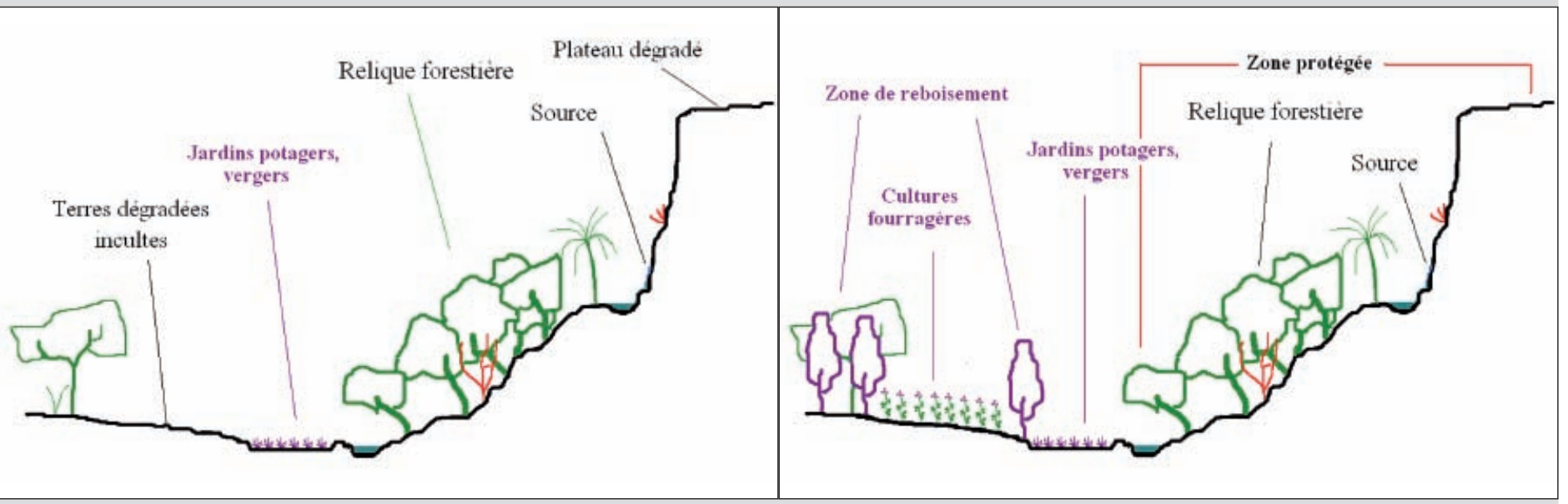

Figure 5.

Propositions d'aménagement de la falaise au niveau de Dafra : à gauche, état actuel ;

à droite, site aménagé.

\section{Les pratiques agricoles}

En agriculture, certaines pratiques de travail du sol peuvent être très néfastes pour le sol et sa fertilité. Des techniques adéquates comme l'aménagement de bandes antiérosives seront privilégiées pour limiter les risques d'érosion (photo 18). Il est prudent d'éviter de labourer les bordures du champ ou les rigoles d'érosion. Un dispositif antiérosif léger tel que des branchages qui protègent le repiquage d'Andropogon gayanus Kunth (Poaceae) peut être efficace (photo 20).

Les graves dégâts provoqués par le labour des berges, après disparition des galeries, peuvent être simplement combattus en conservant une bande enherbée de 4 à $6 \mathrm{~m}$ de large le long du cours d'eau, en conservant ou en favorisant la végétation arbustive ou en plantant des cultures telles que le palmier à huile ou le bananier (figure 4). Si le producteur est convaincu de l'utilité de ces mesures, le remède est simple à appliquer et à la portée de tous.

\section{L'exploitation du bois et des ressources forestières}

L'approvisionnement de la ville de Bobo-Dioulasso en bois de chauffe se fait au détriment des formations végétales naturelles. Les ressources forestières, surexploitées, s'amenuisent de jour en jour et satisfont de moins en moins facilement la demande énergétique croissante de cette ville à l'évolution démographique rapide. La plantation d'arbres est une solution tout à fait réaliste car une partie de l'espace rural est devenue disponible pour le reboisement: au pied de la falaise, une mauvaise gestion des terres a rendu de grandes surfaces impropres à l'agriculture et même à l'élevage (figure 4). La recherche a, depuis de nombreuses années, identifié des espèces autochtones ou exotiques aptes aux reboisements et a étudié leur sylviculture.

Parmi les exotiques à croissance initiale rapide que l'on doit réserver aux terrains dégradés des zones agraires, entre autres il est conseillé Eucalyptus camaldulensis Dehn. (Myrtaceae) qui fournit non seulement du bois de feu mais aussi des produits plus rémunérateurs comme le bois de ser- 
vice et les poteaux. Acacia auriculiformis A. Cunn. ex-Benth. (Mimosaceae), qui aurait pour avantage de restaurer rapidement la fertilité des sols, a été testé ; mais cette espèce est plus sensible à la sécheresse. Dans les zones protégées au pied de la falaise (figure 5), il est préférable de replanter des espèces locales, dont le démarrage est moins rapide et la croissance plus lente, mais dont la longévité est bien supérieure. Khaya senegalensis (Desr.) A. Juss. (Meliaceae), Afzelia africana Sm. exPers. (Caesalpiniaceae), Pterocarpus erinaceus, et d'autres, sont tout à fait aptes à restaurer une forêt plurispécifique sur ces sites à préserver, notamment les zones d'éboulis. En aval, pour restaurer la fertilité des sols qui étaient cultivés, il est possible de semer, après labour, des légumineuses de couverture comme Mucuna pruriens var. utilis (Wall. ex-Wright) Bak. ex-Burck (Fabaceae).

Les questions majeures qui se posent alors sont « comment réaliser les plantations ? », « comment les exploiter ensuite de manière collective ou individuelle? " et « comment faire participer les organisations féminines qui exploitent aujourd'hui le bois des forêts naturelles même dégradées ? ». La réponse est peut-être entre les mains des associations écologiques locales qui, à l'exemple du Green Belt Movement au Kenya, peuvent, par la sensibilisation et la mobilisation ${ }^{1}$, avoir un impact rapide et visible sur l'environnement et la production de bois.

\section{L’exploitation minière du sable et du gravier}

La pratique de l'exploitation minière est de loin la plus dangereuse pour les ressources naturelles. La coupe des arbres est grave, certains par chance peuvent souvent rejeter de souche et favoriser le reboisement. La destruction du sol, par contre, rend la surface irrémédiablement improductive. Cette menace est d'autant plus insidieuse que les chantiers d'exploitation sont souvent de taille réduite et très disséminés. Ils disparaissent dans le paysage et peuvent passer inaperçus à un visiteur non averti. Les camions sillonnent tous les niveaux de la falaise. Vers Koro et Kouakoualé, les traces d'extraction sont présentes partout. Encore une fois, tout comme le labour sans contrainte, ce sont les pratiques humaines anarchiques qui sont en cause et non les besoins en matériaux. Car l'exploitation pourrait être circonscrite à une carrière bien délimitée avec infiniment moins de dégâts sur les ressources naturelles.

Le remède est ici très simple, en apparence. Il suffirait de délimiter des zones d'exploitation. En réalité, comme le chargement d'un camion est payé de 5000 à 20000 francs Cfa au propriétaire terrien (BOUCARD, 2007), ce complément de revenus est tentant d'autant plus qu'il n'est pas nécessaire d'en référer à une quelconque autorité.

${ }^{1}$ Le Green Belt Movement est une association créée par Wangari Maathai, prix Nobel de la Paix 2004. Sa vision est de créer une société fondée sur des valeurs humanistes pour améliorer les conditions de vie dans un environnement plus « vert » et plus équitable. Depuis 1977, au Kenya, le reboisement par les femmes, dans les terres agricoles, a considérablement modifié les paysages et a grandement résolu les problèmes de bois énergie et d'érosion sur des milliers d'hectares. 


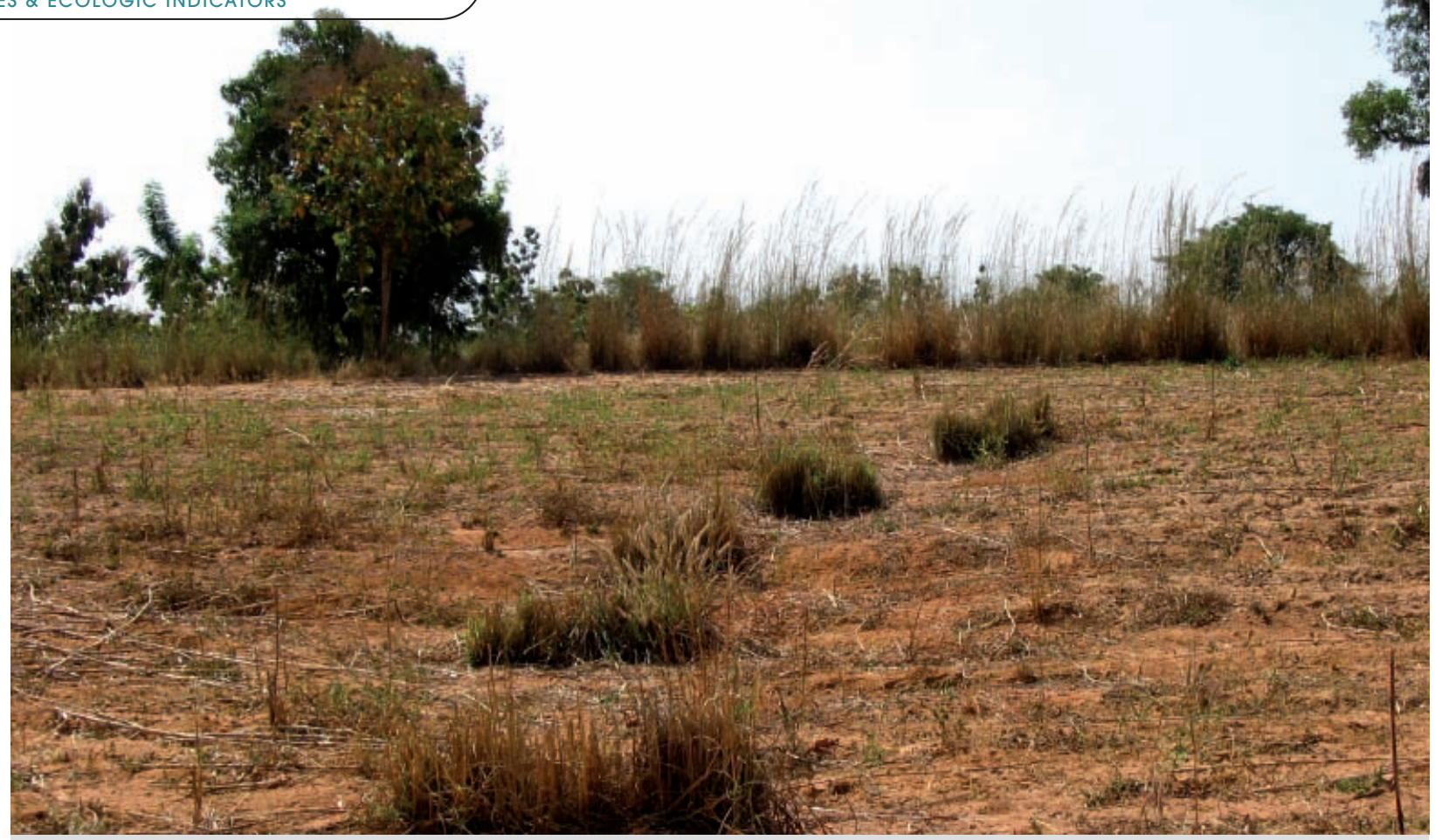

Photo 20.

Dispositif antiérosif dans le même champ que celui de la photo 17 , après deux années de culture de sorgho.

Photo J. César.

pas le problème de l'exploitation minière sur les terres non cultivables, telles que le piémont des falaises. Des plantations d'arbres, même d'eucalyptus, pourraient être une solution.

L'extraction minière ne se limite pas à la falaise. Au pied de la falaise, l'extraction du sable a eu pour conséquence la reprise d'érosion régressive dans la plaine alluviale de Koro, et le creusement d'un nouveau lit. Cette érosion dangereuse a déjà détruit plusieurs champs et compromet l'avenir des cultures maraîchères et fruitières dans la plaine. Les autorités administratives ou traditionnelles sont impuissantes devant les intérêts individuels car les cadres de concertation sont inexistants ou inopérants.

Coupe du bois, érosion, extraction de sol, ces dégradations sont plus fortes à Koro et Kouinima qu'ailleurs, par suite de la proximité de la ville. Faut-il pour autant renoncer à conserver un patrimoine écologique, culturel, forestier et agronomique qui nourrit en grande partie la ville de Bobo-Dioulasso?

Les dégâts sont graves. L'ampleur des phénomènes dépasse le cadre des quelques villages qui entourent BoboDioulasso. L'évolution est rapide, exponentielle. Des mesures urgentes sont à prendre si le souhait est de préserver ce qui reste des ressources et sécuriser les producteurs de ces terroirs aménagés pour la production maraîchère et fruitière.

Quelques remèdes ont été évoqués ; il en reste sans doute beaucoup d'autres. Dans cet état d'urgence, il convient de hiérarchiser les interventions. Elles devront se faire dans l'ordre suivant:

1. arrêter l'exploitation minière anarchique ;

2. protéger et régénérer les galeries forestières ;

3. récupérer des terres incultes pour la production de bois et de fourrage ;
4. améliorer la productivité individuelle par la diffusion de plantes performantes et améliorantes, ainsi que les itinéraires techniques ;

5. lutter contre l'érosion dans les champs.

Ces mesures sont certainement insuffisantes, mais déjà leur introduction expérimentale dans le cadre des activités du Projet Fsp 2002-87 «Gestion durable des ressources sylvo-pastorales et production fourragère dans l'Ouest du Burkina-Faso » (CIRDES, 2009 ; CÉSAR et al., $2008,2010)$ semble avoir convaincu une partie des villageois de Koro, Kouakoualé et Kuinima.

Des mesures législatives sont peut-être à prendre, mais le plus important est de faire respecter les règles qui existent déjà. Il convient de délimiter des zones d'exploitation du sable, des ressources ligneuses ou pastorales, et donner les autorisations adéquates permettant l'exploitation raisonnée de ces ressources. Il est indispensable aussi de compléter une sensibilisation des populations et des exploitants, déjà bien apprise par cœur, mais peu efficace. On le voit, il reste encore beaucoup à faire.

\section{Remerciements}

Les auteurs remercient l'Ambassade de France à Ouagadougou pour le financement du Projet Fsp 2002-87 "Gestion durable des ressources sylvo-pastorales et production fourragère dans l'Ouest du Burkina-Faso », qui a permis de réaliser cette étude, ainsi que Jakob Fahr et Natalie Weber (Université d'Ulm, Allemagne) pour leur aide dans l'étude des chauves-souris. 


\section{Références bibliographiques}

AKOUDJIN M., 2011. Évaluation de l'état écologique des écosystèmes savanicoles et reliques forestières de l'ouest du Burkina Faso à partir des insectes bio-indicateurs de la famille des Nymphalidae et des Cetoniinae. Sarrebruck, Allemagne, Éditions universitaires européennes, 72 p.

AKOUDJIN M., CÉSAR J., KOMBASSERE A., BOUYER J., 2011. Spatio-temporal variability of fruit feeding insects used as ecological indicators in West Africa. Bois et Forêts des Tropiques, 308 (2) : dans ce numéro.

BOUCARD A., 2007. L'exploitation des ressources naturelles dans les stratégies de sécurité alimentaires des ménages. Enquêtes socio-économiques auprès des acteurs de la filière informelle de bois énergie dans la région de BoboDioulasso. Master Développement agricole durable : économie internationale et sécurité alimentaire, Université Paris Sud, Faculté Jean Monnet, France, 57 p.

BOUYER J., GUERRINI L., CÉSAR J., DE LA ROCQUE S., CUISANCE, D., 2005. A phyto-sociological analysis of the distribution of riverine tsetse flies in Burkina Faso. Medical and Veterinary Entomology, 19: 372-378.

BOUYER J., SANA Y., SAMANDOULGOU Y., CÉSAR J., GUERRINI L., KABORE-ZOUNGRANA C., DULIEU D., 2007. Identification of ecological indicators for monitoring ecosystem health in the trans-boundary W Regional park: a pilot study. Biological Conservation, 138: 73-88.

CÉSAR J., AKOUDJIN M., BOUYER J., 2008. Mutations agrosylvo-pastorales autour de Bobo-Dioulasso et de la falaise de Banfora. Atelier régional « Changements climatiques et interactions élevage et environnement en Afrique de l'Ouest », Niamey, 11-15 février 2008, 8 p.

CÉSAR J., BOUYER J., GRANJON L., AKOUDJIN M., 2008. Les dégradations autour de Bobo-Dioulasso et sur la falaise de Banfora. Cirdes, Projet Fsp 2002-87 « Gestion durable des ressources sylvo-pastorales et production fourragère dans l'Ouest du Burkina-Faso », fiche technique, 20 p.

CÉSAR J., BOUYER J., GRANJON L., AKOUDJIN M., GUERRINI L., LOUPPE D., 2010. Les relictes forestières de la falaise de Banfora, un peuplement original au voisinage de BoboDioulasso, Burkina Faso. Bois et forêts des tropiques, 305 (3) : 43-55.

CÉSAR J., KANWE A., ZONGO L, AKOUDJIN M., 2009. Expérimentations en cultures fourragères dans la région de BoboDioulasso. In : César J., Kanwe A., Gouro A., Dicko M., Ehouinsou M., Aboh A. B., Desquesnes M., Zongo L., Akoudjin $M$. Production fourragère au Cirdes et dans la région de Bobo-Dioulasso. Cirdes, Cirad, Ppzs, 1 CD.

CÉSAR J., ZOUMANA C., 1999. Les régimes alimentaires des bovins, ovins et caprins dans les savanes de Côte-d'Ivoire, et leurs effets sur la végétation. Fourrages, 159 : 237-252.

CHEVALLIER G., 1994. Caractérisation agro-sylvo-pastorale et utilisation des pâturages par les éleveurs en saison des pluies. Le cas de Kourouma au Burkina Faso. Mémoire de fin d'études, Cnearc, Montpellier, France, 91 p. + annexes.
CIRDES, 2009. Gestion durable des ressources sylvo-pastorales et production fourragère dans l'Ouest du BurkinaFaso. Projet Fsp 2002-87, 1 CD.

DAGET P., POISSONET J., 1971. Une méthode d'analyse phytologique des prairies. Critères d'application. Annales Agronomiques, 22 (1) : 5-41.

DE LA ROCQUE S., DIALLO M., CÉSAR J., 2004. Analysis of the anthropic causes of changes in riverbank formations in an agropastoral area of Burkina Faso (Sidéradougou). In: Babin D. (éd.). Beyond Tropical Deforestation. Paris, France, Unesco-Cirad, p. 201-203.

DELPECH R., 1960. Critères de jugement de la valeur agronomique des prairies. Fourrages, 4 : 83-98.

DUPLANTIER J.-M., 1998. Les petits rongeurs indicateurs des modifications du climat, des milieux et des pratiques agricoles dans la vallée du fleuve Sénégal. In : Hervé J.-P., Brengues J. (éds). Aménagements hydro-agricoles et santé. Montpellier, France, Ird Éditions, p. 53-65.

FAHR J., 2011. Bats of West Africa. In: Kampmann D., Thiombiano A. (eds). Biodiversity Atlas of West Africa, Vol. 1: Burkina Faso. Sous presse.

GRANJON L., DUPLANTIER J.-M., 2009. Les rongeurs de l'Afrique sahélo-soudanienne. Montpellier, France, Ird Éditions, coll. Faune et flore tropicales, $216 \mathrm{p}$.

GRANJON L., HOUSSIN C., LECOMPTE E., ANGAYA M., CÉSAR J., CORNETTE R., DOBIGNY G., DENYS C., 2004. Community ecology of the terrestrial small mammals of Zakouma National Park, Chad. Acta Theriologica, 49: 215-234.

GUERRINI L., BORD J.-P., DUCEYNE E., BOUYER J., 2008. Fragmentation analysis for prediction of suitable habitat for vectors: the example of riverine tsetse flies in Burkina Faso. Journal of Medical Entomology, 45: 1180-1186.

KALKO E., FAHR J., 2011. Sampling bats. In: Kampmann D., Thiombiano A. (eds). Biodiversity Atlas of West Africa, Vol. 1: Burkina Faso. Sous presse.

KANGOYÉ M. N., THIOMBIANO A., GRANJON L., KALKO E. K. V., FAHR J. (IN PREP.). Diversity and distribution of bats (Chiroptera) from Burkina Faso.

KIEMA S., FOURNIER A., 2007. Utilisation de trois aires protégées par l'élevage extensif dans l'Ouest du Burkina Faso. In : Fournier A., Sinsin B., Mensah G. A. (eds). Quelles aires protégées pour l'Afrique de l'Ouest ? Conservation de la biodiversité et développement. Paris, France, Ird Éditions, coll. Colloques et séminaires, $607 \mathrm{p}$.

MEYER C. F. J., AGUIAR L. M. S., AGUIRRE L. F., BAUMGARTEN J., CLARKE F. M., COSSON J.-F., ESTRADA VILLEGAS S., FAHR J., FARIA D., FUREY N., HENRY M., HODGKISON R., JENKINS R. K. B., JUNG K. G., KINGSTON T., KUNZ T. H., MACSWINEY GONZALEZ M. C., MOYA I., PONS J.-M., RACEY P. A., REX K., SAMPAIO E. M., STONER K. E., VOIGT C. C., VON STADEN D., WEISE C. D., KALKO E. K. V., 2010. Long-term monitoring of tropical bats for anthropogenic impact assessment: gauging the statistical power to detect population change. Biological Conservation, 143: 2797-2807. 